\title{
DESCRIPCIÓN DE LOS ESTADIOS NINFALES DE CEBRENISCELLA ANTENNATA BRAILOVSKY (HEMIPTERA: HETEROPTERA: COREIDAE: COREINAE: COREINI), SU DISTRIBUCIÓN EN MÉXICO Y NOTAS SOBRE SU BIOLOGÍA
}

\author{
JEZABEL BÁEZ SANTACRUZ ${ }^{1}$ y LUIS CERVANTES PEREDO ${ }^{1 \dagger}$ \\ ${ }^{1}$ Red de Biodiversidad y Sistemática, Instituto de Ecología, A.C. Carretera Antigua a Coatepec, No. 351, El Haya, \\ Xalapa, C.P. 91070. Veracruz. México <jezabel.baez@inecol.mx>
}

Recibido: 24/11/2014; aceptado: 04/03/2015

Báez, J. \& Cervantes, L. 2015. Descripción de los estadios ninfales de Cebreniscella antennata Brailovsky (Hemiptera: Heteroptera: Coreidae: Coreinae: Coreini), su distribución en México y notas sobre su biología. Acta Zoológica Mexicana (n. s.), 31(2): 265269.

RESUMEN. Se describen e ilustran en detalle todos los estadios de Cebreniscella antennata Brailovsky, incluyendo notas sobre su biología, se le ha encontrado alimentándose de las estructuras reproductoras de varias especies de Vernonia (Asteraceae), y se encuentra distribuida desde el estado de Jalisco, hasta Oaxaca, incluyendo Morelos y el Estado de México.

Palabras Clave: Estadios, Compositae, flores.

\section{INTRODUCCIÓN}

El género Cebreniscella fue descrito por Brailovsky (1995), basado en la especie Cebrenis exitiosa Brailovsky (1984), e incluyendo también a Cebreniscella antennata Brailovsky (1995). Este género muy similar al género Cebrenis Stål, 1862, se diferencia por el aspecto particular de los genitales de ambos sexos. El esternito VII y las placas genitales de la hembra de Cebreniscella están ensanchados y nunca lateralmente aplanados o pegados a la gonocoxa I, como en Cebrenis. Los parámeros de los machos de Cebreniscella son largos, delgados y con el tercio apical en forma de mazo; mientras que en Cebrenis nunca son alargados.

El género Cebrenis está conformado por 23 especies, divididas en 4 grupos y con distribución principalmente Sudamericana, con solo C. danieli Brailovsky en México; mientras que Cebreniscella contiene 2 especies, ambas en México, aunque C. exitiosa (Brailovsky) también se distribuye en Costa Rica.

Del complejo Cebrenis, que incluyen los géneros Cebrenis, Cebreniscella y Cebrenistella, sólo se tienen registros de las plantas hospederas de algunas especies y no se han descrito los estados inmaduros de ninguna especie.

Son pocos los trabajos que describen estados inmaduros de coreidos (Brailovsky et al. 1994, 1995) y en especial de la Tribu Coreini (Brailovsky et al. 1998; Chacón et al. 2012; Báez \& Cervantes 2014).
Báez, J. \& Cervantes, L. 2015. Description of the immature stages of Cebreniscella antennata Brailovsky (Hemiptera: Heteroptera: Coreidae: Coreinae: Coreini), its distribution in Mexico and notes on its biology. Acta Zoológica Mexicana (n. s.), 31(2): 265-269.

ABSTRACT. Descriptions and illustrations of all instars about $\mathrm{Ce}$ breniscella antennata Brailovsky, and notes on its biology, it has been found feeding on reproductive structures of several species of Vernonia (Asteraceae), and it is distributed from Jalisco to Oaxaca, including Morelos and the State of Mexico.

Key Words: Instars, Compositae, flowers

En el presente trabajo se describen e ilustran en detalle, por primera vez, todos los estadios de Cebreniscella antennata, incluyendo notas acerca de su biología, plantas hospederas y registros de su distribución en México.

\section{MATERIAL Y MÉTODOS}

Para el presente trabajo se utilizaron ejemplares depositados en la Colección Nacional de Insectos, Universidad Nacional Autónoma de México (CNIN) y en la Colección Entomológica del Instituto de Ecología, A. C. Xalapa Veracruz (IEXA).

Los ejemplares utilizados para la cría se obtuvieron en la localidad de Putla de Guerrero, Km 15 Carretera Putla-Tlaxiaco, en el estado de Oaxaca, a $17^{\circ} 03^{\prime} 41^{\prime}$ 'N, 9752'11'’W, 556 m snm, con vegetación predominante de Bosque de Pino-Encino. Los ejemplares se colectaron utilizando aspirador. Los adultos se depositaron en contenedores de plástico $(9 \times 8 \mathrm{~cm})$. Se colocó en cada contenedor dos o tres semillas de girasol y un algodón humedecido, cada tres días se revisaron los contenedores, se cambiaron las semillas y se registró la presencia de huevos, eclosión, muda o muerte de los ejemplares. Las ilustraciones se realizaron con ayuda de un microscopio Leica MZ8 adaptado con un tubo de dibujo. Las medidas están expresadas en milímetros \pm una desviación estándar. 


\section{Cebreniscella antennata Brailovsky}

(Figs. 1-7)

1995. Cebreniscella antennata Brailovsky, Pub. Esp. Univ. Nac. Aut. Mex. 15: 115-119.

Huevo (Fig. 1): Ovalado, con la parte superior plana y la inferior ligeramente redondeada. Mide $0.94 \pm 0.08 \mathrm{~mm}$ de largo por $0.81 \pm 0.06 \mathrm{~mm}$ de ancho $(n=10)$. De color blanquecino al ser depositados, tornándose pardo claro posteriormente. Parte superior del huevo más clara. Superficie ligeramente reticulada.

Primer estadio (Fig. 2): Piriforme. Coloración general rojiza. Cabeza, pro-, meso- y metanoto pardo claro con una línea longitudinal submarginal roja; márgenes laterales hialinos marcados con una línea negra marginal; cabeza con una banda longitudinal clara, apenas visible, desde el tylus sin llegar a la base de la cabeza; ojos rojos y sésiles; rostro amarillo pálido con ápice pardo obscuro; tubérculo antenífero con ápice rojizo; antenas rojizas con uniones intersegmentales blancas; línea de dehiscencia más ancha en meso- y metanoto, demarcada con una línea lateralmente roja y a cada lado con una banda más clara; pronoto con márgenes anterior y posterior color pardo obscuro; pleuras con una banda ancha basal color rojizo; patas color amarillo pálido; fémur y tibia con tres hileras longitudinales de granulaciones setígeras rojas; abdomen rojizo, rodeado por una línea roja; placas de las glándulas senescentes ubicadas en los segmentos IV-V y V-VI pardo obscuras, rodeadas por un mancha rojiza.

Cabeza alargada, de igual longitud que el tórax; tylus rebasando ligeramente los juga, ápice redondeado. Rostro alcanzando el ápice de metacoxa. Pronoto con margen superior ligeramente cóncavo y posterior, recto. Artejos antenales cubiertos de sedas largas, gruesas y negras, principalmente en aristas; cuatro sedas prominentes en ápice de artejos II y III; IV con ápice pubescente; artejo antenal I corto, robusto y cuadrangular; II y III aplanados, con dos lados y con aristas pardo obscuras; IV fusiforme. Tylus con dos hileras de sedas que convergen en una, llegando a la línea de dehiscencia; base del tubérculo antenífero con dos; 3-4 cercanas al margen interno del ojo y dos cercanas a la base; $4-5$ sedas cercanas a la base de cabeza. Pronoto con el margen superior ligeramente cóncavo y el posterior recto; pro-, meso- y metanoto con 3-5 sedas en márgenes laterales, submargen con dos y tres a cada lado de la línea de dehiscencia. Segmentos abdominales I-VII con una línea transversal de 6-8 sedas cortas en el margen anterior y posterior; segmento IX con dos sedas largas. Segmentos abdominales I-VII con un par de espiráculos, pardos obscuros, provistos de un tribobotrio grueso y negro.

Mediciones $(n=6)$. Longitud total del cuerpo $2.34 \pm 0.13$; longitud de la cabeza $0.66 \pm 0.07$; anchura a través de los ojos $0.52 \pm 0.03$; distancia interocular $0.36 \pm 0.04$; distan- cia postocular $0.17 \pm 0.03$; artejos antenales: I $0.3 \pm 0.03$, II $0.52 \pm 0.03$, III $0.54 \pm 0.05$, IV $0.4 \pm 0.03$; artejos rostrales: I $0.4 \pm 0.04$, II $0.41 \pm 0.05$, III $0.22 \pm 0.03$, IV 0.39 \pm 0.02 ; longitud del pronoto $0.17 \pm 0.03$; anchura ángulos humerales $0.5 \pm 0.03$; anchura a través del margen anterior del pronoto $0.5 \pm 0.03$; pata posterior: longitud fémur $0.51 \pm 0.02$; longitud tibia $0.48 \pm 0.04$; longitud tarsos: I 0.15 , II $0.21 \pm 0.04$.

Segundo estadio (Fig. 3): Forma y coloración similar al estadio I. Antenas, ojos, cabeza, pro-, meso- y metanoto color pardo claro; patas ocre claro, fémur ligeramente más obscuro que la tibia; tarso II con ápice negro; dorso abdominal ligeramente rojizo con puntos rojos pequeños, dispersos, apenas visibles; dos bandas longitudinales submarginales rojizas desde el segmento I, desvaneciéndose y convergiendo en el VII; base de III-VIII marcados por una línea roja transversal; ventralmente, ocre con puntos rojos pequeños; dos bandas medias longitudinales rojizas. Ápice del tylus y juga redondeado. Rostro alcanzando el ápice del segmento abdominal I.

Mediciones $(n=3)$. Longitud total del cuerpo $2.57 \pm 0.06$; longitud de la cabeza $0.78 \pm 0.03$; anchura a través de los ojos $0.58 \pm 0.03$; distancia interocular $0.48 \pm 0.03$ distancia postocular 0.2 ; artejos antenales: I 0.5 , II $0.63 \pm$ 0.12 , III $0.57 \pm 0.06$, IV $0.43 \pm 0.06$; artejos rostrales: I 0.4 , II $0.37 \pm 0.06$, III 0.21 , IV 0.3 ; longitud del pronoto 0.25 ; anchura ángulos humerales $0.58 \pm 0.03$; anchura a través del margen anterior del pronoto $0.58 \pm 0.03$; pata posterior: longitud fémur $0.57 \pm 0.06$; longitud tibia 0.57 \pm 0.06 ; longitud tarsos: I $0.17 \pm 0.03$, II 0.2 .

Tercer estadio (Fig. 4): Piriforme. Coloración general ocre a rojiza. Cabeza y antenas de color pardo claro; ápice de artejos antenales rojos; ventralmente, lados de la cabeza corre una banda longitudinal parda rojiza, la cual pasa por la base de las pleuras alcanzando los segmentos abdominales y desvaneciéndose en los segmentos VIII y IX; pronoto y metanoto pardo claros con una banda longitudinal más obscura cercana a la línea de dehiscencia, hasta alcanzar la base del segmento abdominal III, donde se torna roja; márgenes laterales del pro- y mesonoto aserrados con sedas cortas y con una línea parda obscura; patas ocres; ápice del tarso II pardo obscuro; fémur y tibia con granulaciones setígeras pardas, alineadas en hileras longitudinales; segmentos abdominales V y VI con la base color rojo; placas de las glándulas senescentes color pardo obscuro y rodeadas por una mancha anaranjada clara.

Cabeza alargada con una longitud mayor que el pronoto; artejo antenal I ligeramente curvado hacia afuera; II y III con aristas rojas. Rostro alcanzando el ápice de metacoxa. Paquetes alares alcanzando la base del segmento abdominal I. Pronoto con pocas sedas cortas y espaciadas; 

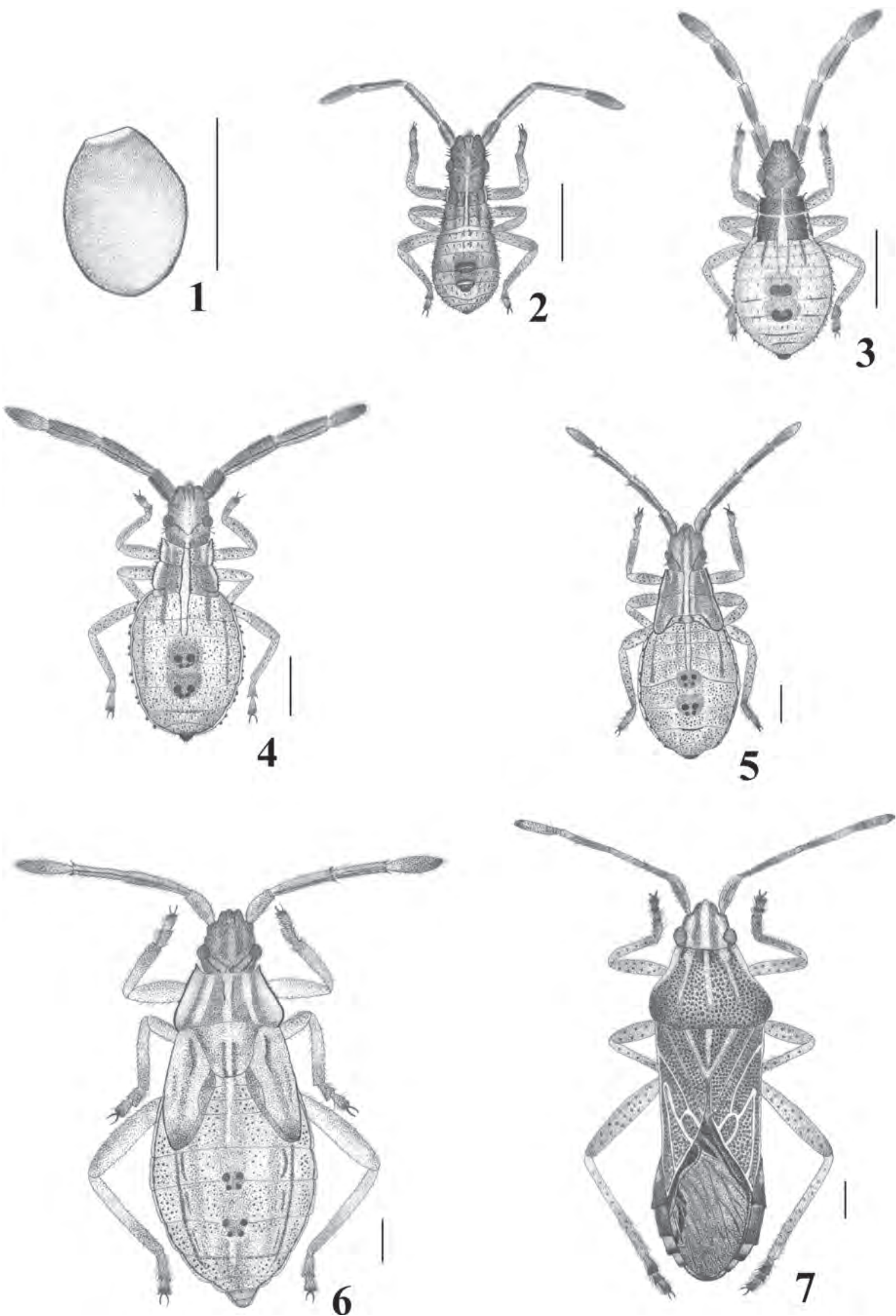

Figuras 1-7. Estadios del desarrollo de Cebreniscella antennata Brailovsky. 1. Huevo vista lateral. 2. Primer Estadio. 3. Segundo Estadio. 4. Tercer Estadio. 5. Cuarto Estadio. 6. Quinto Estadio. 7. Adulto 
margen anterior ligeramente cóncavo y posterior recto; ángulos frontales salientes y agudos. Abdomen con sedas cortas y apenas visibles en los segmentos I-VII, y más largas en los segmentos VIII y IX.

Mediciones $(n=6)$. Longitud total del cuerpo $3.78 \pm 0.22$; longitud de la cabeza $0.95 \pm 0.06$; anchura a través de los ojos $0.78 \pm 0.11$; distancia interocular $0.55 \pm 0.08$; distancia postocular $0.19 \pm 0.02$; artejos antenales: I 0.48 \pm 0.04 , II $0.83 \pm 0.05$, III $0.77 \pm 0.02$, IV $0.69 \pm 0.02$; artejos rostrales: I $0.67 \pm 0.04$, II $0.62 \pm 0.03$, III $0.31 \pm$ 0.02 , IV $0.52 \pm 0.07$; longitud del pronoto $0.32 \pm 0.09$; anchura ángulos humerales $0.87 \pm 0.07$; anchura a través del margen anterior del pronoto $0.74 \pm 0.04$; pata posterior: longitud fémur $0.73 \pm 0.05$; longitud tibia $0.68 \pm 0.07$; longitud tarsos: I $0.24 \pm 0.04$, II $0.28 \pm 0.04$.

Cuarto estadio (Fig. 5): Forma y coloración similar al estadio III. Cabeza pardo claro, ligeramente más obscura a los lados; margen interno del ojo con una línea roja, que se extiende a la línea de dehiscencia; puntuaciones pequeñas, pardas y dispersas en cabeza y artejos antenales; ápice de artejos antenales II y III con dos manchas negras, sedas negras y gruesas; pronoto y paquetes alares pardo claros con bandas longitudinales, blanquecinas y discontinuas; márgenes laterales con una banda ocre y continua, seguida por una línea negra; lados de la línea de dehiscencia con una banda blanquecina, desde el margen anterior del pronoto a la base del segmento abdominal VIII. Abdomen ocre con puntos rojos apenas visibles; segmentos I y II con dos bandas medias longitudinales color rojo; dos franjas rojas submarginales entre los segmentos I-VII, aclarándose en los últimos segmentos; segmentos III-VII con una línea transversal roja muy delgada en la base.

Superficie dorsal con algunas sedas, más cortas y claras que en los estadios anteriores. Tubérculo antenífero con una proyección corta y apicalmente roma; base del tubérculo con tres sedas prominentes. Tylus conserva la línea longitudinal de sedas; base de ojos con dos sedas cortas. Rostro alcanzando la base del segmento abdominal I. Margen anterior del pronoto, cóncavo y posterior ligeramente convexo. Paquetes alares rebasando la mitad del segmento abdominal II.

Mediciones $(n=10)$. Longitud total del cuerpo $5.74 \pm$ 0.23 ; longitud de la cabeza $1.19 \pm 0.09$; anchura a través de los ojos $0.99 \pm 0.03$; distancia interocular $0.67 \pm 0.05$; distancia postocular 0.2; artejos antenales: I $0.62 \pm 0.07$, II $1.08 \pm 0.09$, III $0.95 \pm 0.05$, IV $0.8 \pm 0.08$; artejos rostrales: I $0.75 \pm 0.06$, II $0.72 \pm 0.05$, III $0.42 \pm 0.03$, IV $0.72 \pm 0.04$; longitud del pronoto $0.56 \pm 0.05$; anchura ángulos humerales $1.41 \pm 0.06$; anchura a través del margen anterior del pronoto $1.02 \pm 0.06$; longitud total del escutelo $0.56 \pm 0.06$; anchura máxima de escutelo $0.74 \pm$ 0.05 ; pata posterior: longitud fémur $1.1 \pm 0.09$; longitud tibia $1.03 \pm 0.07$; longitud tarsos: I $0.24 \pm 0.05$, II $0.31 \pm$ 0.03 .

Quinto estadio (Fig. 6): Coloración general ocre. Cabeza con dos franjas color pardo claro con granulaciones pequeñas y pardas, desde los juga pero sin llegar a base de cabeza; ojos y ocelos rojos; pronoto con dos bandas anchas longitudinales, pardas y una blanquecina, longitudinales con puntuaciones pequeñas; márgenes laterales poco aserrados; escutelo con dos bandas longitudinales negras; paquetes alares alcanzando el margen anterior del segmento abdominal IV, ápice de las almohadillas alares más obscuro, margen lateral con una línea delgada negra, y dos franjas longitudinales pardas discontinuas; abdomen con varios puntos rojos pequeños, franja pardo obscura longitudinal, cercana al margen lateral, visible en los segmentos abdominales IV-VII; línea roja que rodea el abdomen apenas visible.

Superficie dorsal con algunas sedas apenas visibles. Cabeza con una seda corta en base del ojo y el tylus con algunas sedas delgadas. Antenas y tarsos pubescentes. Fémures y tibias con puntuaciones rojizas o pardas; tarso II con el ápice ligeramente obscurecido. Rostro alcanzando la base del segmento abdominal I.

Mediciones $(n=10)$. Longitud total del cuerpo $8.01 \pm$ 0.52 ; longitud de la cabeza $1.43 \pm 0.13$; anchura a través de los ojos $1.43 \pm 0.05$; distancia interocular $0.94 \pm 0.05$; distancia entre ocelos $0.47 \pm 0.05$; distancia postocular $0.2 \pm 0.07$; artejos antenales: I $0.99 \pm 0.06$, II $1.53 \pm 0.12$, III $1.27 \pm 0.08$, IV $1.18 \pm 0.06$; artejos rostrales: I $1.02 \pm$ 0.08 , II $1.06 \pm 0.08$, III $0.58 \pm 0.08$, IV $0.92 \pm 0.06$; longitud del pronoto $1.17 \pm 0.09$; anchura ángulos humerales $2.32 \pm 0.11$; anchura a través del margen anterior del pronoto $1.34 \pm 0.07$; longitud total del escutelo $1.04 \pm 0.14$; anchura máxima de escutelo $1.24 \pm 0.1$; pata posterior: longitud fémur $1.58 \pm 0.14$; longitud tibia $1.41 \pm 0.14$; longitud tarsos: I $0.45 \pm 0.05$, II $0.46 \pm 0.05$.

Adulto (Fig. 7): Coloración dorsal amarillo ocre pálido, con puntuaciones pardo castaño o pardo rojizo y con las siguientes áreas de color crema: cabeza con tres franjas longitudinales, una media y las otras dos corriendo entre el ojo y el ocelo, y cada una de ellas se continua a través del disco pronotal y del escutelo; los ángulos frontales y los márgenes anterolaterales del pronoto, el ápice del escutelo, las venas claval y coriales y el margen costal y apical del corium también color crema; membrana hemelitral de color humo pálido a casi translucido y con el ángulo basal y una mancha alargada situada entre la cara externa y media de color humo oscuro a casi negro. Artejo antenal I amarillo ocre pálido con reflejos anaranjados y con los gránulos más obscurecidos; artejos antenales II y III anaranjado pálido y el IV amarillo con el tercio basal anaranjado pálido. Coloración ventral amarillo pálido, excepto 
el ápice del artejo rostral IV y una mancha discoidal en la mesopleura, metapleura, mesocoxa, metacoxa y en los esternios abdominales III a VII, que son pardo.

Cabeza con el tubérculo antenífero externamente armado con una proyección corta y apicalmente roma. Artejo antenal I ligeramente carinado; artejos II y III cilíndricos y robustos y IV fusiforme. Miden entre 9.26 y $10.9 \mathrm{~mm}$. Otras características descritas en Brailovsky (1995).

Notas biológicas. Las ninfas y los adultos fueron colectados en Putla, Oaxaca, alimentándose de las flores de Vernonia karvinskiana DC. (Asteraceae). Brailovsky (1995) la registra en Vernonia capreifolia Gleason, en V. aschenborniana S. Schauver.

Distribución. México: Guerrero, Estado de México, Jalisco, Michoacán, Morelos, Oaxaca.

\section{DISCUSIÓN}

Esta es la primera descripción de los estados inmaduros de chinches del complejo Cebrenis, que incluyen los géneros Cebrenis, Cebreniscella y Cebrenistella, y del cual sólo se tenían reportes de las plantas hospederas de algunas especies. En general, podemos decir que las especies de este grupo están asociadas a plantas de la familia Asteraceae, principalmente de los géneros Neurolaena R.Br., Mikania F.W. Schmidt, Verbesina L., Eremosis (DC.) Gleason, Calea L. y Vernonia Schreb. (Brailovsky 1995), alimentándose de las estructuras reproductoras, tanto inflorescencias como de las infrutescencias cuando todavía están adheridas a la planta.

Las ninfas de Cebreniscella antennata se caracterizan por presentar los segmentos antenales II y III aplanados dorsoventralmente, a diferencia de las ninfas de otros miembros de la Tribu Coreini de donde se conocen las ninfas, cuyos segmentos antenales II y III son generalmente cilíndricos. La línea de dehiscencia o muda en las ninfas de $C$. antennata está muy bien marcada en cabeza, tórax y en los primeros 3 segmentos abdominales; y sin embargo en otras especies de Coreini la línea de dehiscencia solo está bien marcada en cabeza y tórax (Brailovsky et al. 1998; Chacón et al. 2012; Báez \& Cervantes 2014; Cervantes et al. 2014).

AGRADECIMIENTOS. Agradecemos al Dr. Harry Brailovsky del Instituto de Biología (UNAM), por la determinación de los ejemplares. Parte del material fue colectado con financiamiento de los siguientes proyectos: DGAPA (IN223503-3) “Análisis comparativo de la coreidofauna en tres estados de la República Mexicana: Guerrero, Oaxaca y Chiapas (Hemiptera-Heteroptera)”.

\section{LITERATURA CITADA}

Báez, J. \& Cervantes, L. 2014. Estados inmaduros y fenología de Catorhintha apicalis scrutator (Hemiptera: Heteroptera: Coreidae) en Michoacán, México. Revista de Biología Tropical, 62: 579-587.

Brailovsky. H. 1995. Revisión del complejo Cebrenis (HemipteraHeteroptera-Coreida-Coreinae-Coreini). Publicaciones Especiales Instituto de Biología, Universidad Nacional Autónoma de México, 15: 1-124.

Brailovsky, H., Barrera, E., Mayorga, C. \& Ortega, G. 1994. Estadios ninfales de los Coreidos del Valle de Tehuacán, Puebla. (Hemiptera-Heteroptera) I. Chelinidea stasffilesi, C. tabulata y Narnia femorata. Anales del Instituto de Biología Universidad Nacional Autónoma de México. Serie Zoología, 65: 241-264.

Brailovsky, H., Mayorga, C., Ortega, G. \& Barrera, E. 1995. Estadios ninfales de los Coreidos del Valle de Tehuacán, Puebla, México (Hemiptera-Heteroptera) II. Especies asociadas a huizacheras (Acacia spp.) y mezquiteras (Prosopis spp.): Mozena lunata. Pachylis hector, Savius jurgiosus y Thasus gigas. Anales del Instituto de Biología Universidad Nacional Autónoma de México. Serie Zoología, 66: 57-80.

Brailovsky, H., Ortega, G., Barrera, E. \& Mayorga, C. 1998. Estadios ninfales de los coreidos del Valle de Téhuacan, Puebla, México (Hemiptera-Heteroptera). III. Anasa ruficornis y Scolopocerus uhleri. Anales del Instituto de Biología Universidad Nacional Autónoma de México. Serie Zoología, 69: 53-64.

Chacón, A., Briceño, M. \& Cervantes, L. 2012. Ciclo de vida de Anasa litigiosa (Hemiptera: Heteroptera: Coreidae). Revista Mexicana de Biodiversidad, 83: 432-436. 